\title{
Etude de la thermostabilité des laits concentrés reconstitués
}

\author{
A. LAGAUDE*, B. TARODO DE LA FUENTE** \\ * Ecole Nationale Supérieure Universitaire de Technologie \\ BP 5085, Dakar Fann, Sénégal \\ ** Laboratoire de Technologie alimentaire \\ Centre de Génie et Technologie alimentaires \\ Université des Sciences et Techniques du Languedoc, \\ place E.-Bataillon - 34060 Montpellier, France
}

\begin{abstract}
Résumé
L'étude de la stabilité thermique des laits concentrés reconstitués a été effectuée à partir de poudres du type "High Heat » (H.H.) et " Medium Heat » (M.H.) à $0 \%$ de matières grasses. La thermostabilité a été appréciée par la mesure du temps de coagulation (T.C.) au laboratoire et par examen visuel après stérilisation industrielle. Les modifications dues aux variations de $\mathrm{pH}$, de la force ionique, ainsi qu'à l'addition de calcium, de phosphate ou d'urée ont été notées. L'évolution du calcium ionique, du calcium soluble et du phosphore soluble a été suivie au cours des différents essais.
\end{abstract}

Mots clés : Lait concentré - Lait reconstitué - Thermostabilité.

\section{Introduction}

La préparation de lait concentré non sucré à partir de poudre s'accompagne de différents problèmes dont le plus important est celui de la stabilité du lait au cours de la stérilisation. La déstabilisation du système caséinate lors du traitement thermique n'est pas propre aux laits reconstitués (poudre+eau) ou aux laits recombinés (poudre + matières grasses + eau) mais elle dépend de processus sensiblement différents de ceux que l'on observe pour les laits normaux. Les traitements successifs appliqués au lait lors de la fabrication de poudre (préchauffage, concentration, déshydratation) sont responsables de profondes modifications pouvant aboutir à une absence de similitude entre la thermostabilité du lait concentré reconstitué et celle du lait d'origine. 
La recherche d'une relation entre la composition du lait et sa stabilité thermique n'a pas abouti aux résultats escomptés; seule l'urée présente une bonne corrélation avec la stabilité thermique (SWeetsur et White, 1975; Holt et al, 1978 b ; Fox, 1982). Sweetsur et WHITe (1975) montrent que l'augmentation de la teneur en protéines lors de la concentration et l'élévation de l'acidité au cours du chauffage sont les 2 principaux facteurs contribuant à la diminution de la stabilité thermique des laits concentrés. Si l'influence du pH est établie (Rose, 1962) celle de la force ionique semble limitée (WEEB et al, 1978). Le calcium en solution n'interviendrait qu'indirectement sur la stabilité thermique par son effet dénaturant sur les protéines du sérum, en particulier sur la $\beta$-lactoglobuline (Rose, 1962; WEeB et al., 1978). Une augmentation de la stabilité thermique peut être espérée par l'addition au lait de phosphate qui diminue l'activité du calcium, influence le $\mathrm{pH}$, modifie la force ionique et relève la teneur en phosphore du lait (Fox, 1982 ; HoRnE, 1982). L'urée ajoutée au moment de la reconstitution d'un lait écrémé à $20 \%$ de matières sèches permet aussi d'améliorer la stabilité thermique (Kelly, 1977) mais uniquement pour les laits produits en milieu de saison (Kelly, 1982).

En abordant la thermostabilité des laits concentrés reconstitués non sucrés, nous nous sommes fixés 2 buts : d'une part apprécier dans quelle mesure les différents tests ou paramètres sont utilisables pour déterminer si une poudre est apte à la préparation de laits stérilisés, d'autre part définir l'importance de quelques composés solubles vis-à-vis de la stabilité thermique du lait reconstitué porté à de hautes températures.

\section{Matériel et méthodes}

\section{A. Choix des poudres de lait}

Nous avons retenu pour cette étude 4 poudres de lait écrémé de différentes origines : 2 poudres "High Heat " (H.H.1 et H.H.2) supposées capables de résister à une stérilisation et 2 poudres " Medium Heat » (M.H.3 et M.H.4) destinées à des préparations ne subissant pas de traitement thermique sévère (JENSEN et NIELSEN, 1982).

L'échantillon H.H.1 se distingue par son excellente tenue au chauffage lors de la préparation industrielle de lait concentré non sucré alors que la poudre H.H.2 exige une addition de phosphate disodique élevée pour supporter la stérilisation.

L'échantillon M.H.3 présente lors d'essais au laboratoire une bonne stabilité thermique bien qu'il n'appartienne pas à la catégorie 
des produits stables à la chaleur. Par contre l'échantillon M.H.4 est caractéristique d'un lait instable aux températures élevées.

\section{B. Préparation des échantillons}

Les poudres sont mises en solution dans de l'eau distillée renfermant $0,02 \%$ d'azoture de sodium utilisé comme agent stabilisant. La reconstitution met en œuvre une quantité de poudre à $0 \%$ de matières grasses suffisante pour obtenir une matière sèche (M.S.) de $17,6 \%(\mathrm{~m} / \mathrm{m})$. La dispersion de la poudre est achevée à l'aide d'un homogénéisateur Ultra Turrax (axe 18K). Les laits reconstitués sont pasteurisés en continu à $73^{\circ} \mathrm{C}$ pendant $3 \mathrm{~min}$. Les ajouts (calcium, phosphate, urée) sont éventuellement effectués puis le $\mathrm{pH}$ corrigé à l'aide d'HCl ou de $\mathrm{KOH}$ dilué. Les laits sont laissés au repos une nuit à $+4^{\circ} \mathrm{C}$.

\section{Détermination de la thermostabilité}

L'appréciation de la thermostabilité est basée sur la détermination du temps nécessaire pour détecter visuellement la coagulation du lait à une température donnée (DARLING, 1980). Nous avons effectué le test à $130^{\circ} \mathrm{C} \pm 0,5^{\circ} \mathrm{C}$. Cette température permet d'avoir des temps de coagulation de l'ordre de $25 \mathrm{~min}$ et une précipitation nette des protéines dans les laits reconstitués; la formation d'agrégats visibles est mesurée à cette température avec une précision de l'ordre de $15 \mathrm{sec}$. Chaque mesure est répétée 3 fois pour chaque échantillon et la moyenne correspondante a été utilisée dans les tableaux et les figures.

Les tests de thermostabilité (mesures du temps de coagulation : T.C.) sont faits sur un échantillon de $1 \mathrm{ml}$ dans des tubes de verre de $8 \mathrm{~mm}$ de diamètre intérieur et de $160 \mathrm{~mm}$ de long, fermés par un bouchon à vis doté d'un joint en silicone. Ces tubes sont disposés horizontalement dans le bain d'huile; l'agitation est assuré par un mouvement de va-et-vient ayant une période de $9 \mathrm{sec}$ (fig. 1).

Les essais de stérilisation industrielle sont réalisés sur des laits reconstitués au laboratoire puis conditionnés dans des boîtes de conserve de $410 \mathrm{~g}$. Le traitement thermique industriel $\left(118^{\circ} \mathrm{C}\right.$ pendant $13 \mathrm{~min}$ ) est effectué sur un stérilisateur en continu Food Machinery and Chemical Corporation (capacité 3022 boîtes).

\section{Ultrafiltration}

Nous utilisons une cellule Amicon modèle 402 équipée d'une membrane Diaflo PM 30. L'ultrafiltration est réalisée à $25^{\circ} \mathrm{C}$ sous une pression de 1 bar et met en jeu $100 \mathrm{ml}$ de lait; l'opération est arrêtée quand $20 \mathrm{ml}$ de filtrat sont recueillis. 


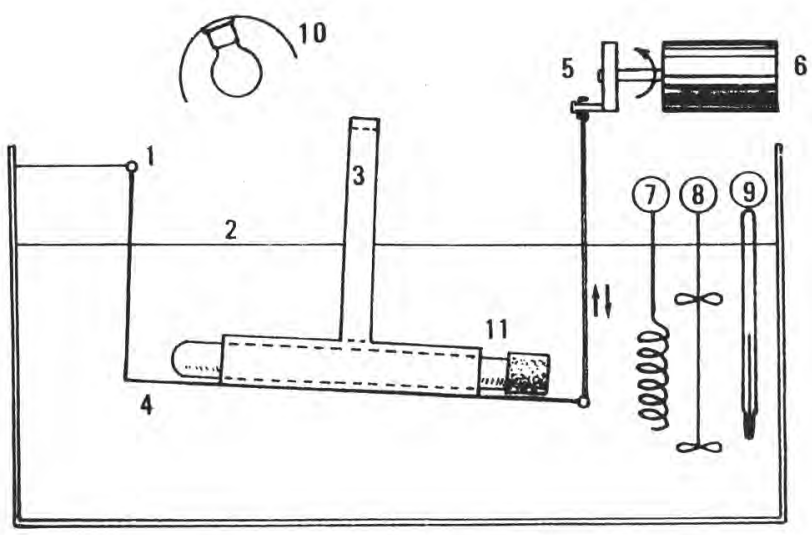

FIG. 1

Système d'agitation des échantillons de lait pour la détermination du temps de coagulation à $130^{\circ} \mathrm{C} .1$ : point fixe ; 2 : niveau $d u$ bain d'huile ; 3 : nacelle amovible en aluminium ; 4 support mobile ; 5 : bielle ; $6:$ moteur $; 7$ : résistance chauffante; 8 : agitateur ; 9 : thermomètre à contact; 10 : éclairage ; 11 tube à essais.

Stirring system of milk samples for coagulation time determination at $130^{\circ} \mathrm{C}$. 1 : settled point ; 2 : aluminium removable nacelle ; 4 : moved support ; 5 : exentric rod; 6 : motor ; 7 : heater resistance ; 8: stirrer ; 9 : electric contact thermometer; 10 : lighting; 11 : test tube.

\section{E. Méthodes d'analyses}

Les matières sèches (M.S.) des poudres et des laits concentrés reconstitués sont déterminées par la méthode préconisée par HaUGAARD SORENSEN et al. (1978).

Les cendres sont obtenues à partir de $3 \mathrm{~g}$ de lait séchés puis incinérés à $530^{\circ} \mathrm{C}$ pendant $3 \mathrm{~h}$.

Le calcium total est évalué par absorption atomique à l'aide d'un appareil Beckman 448 suivant LINDEN (1971); le calcium soluble est dosé par la même technique après ultrafiltration du lait. Le calcium ionique est dosé suivant la méthode de SMEETs complétée par SAINCLIVIER (1959). Le magnésium total et le magnésium soluble sont déterminés par absorption atomique sur les solutions servant au dosage du calcium.

Le sodium, le potassium et le phosphore sont dosés suivant SERres et al. (1973). Le phosphore soluble est dosé directement sur l'ultrafiltrat suivant la même méthode.

L'azote total des laits reconstitués à 17,6\% M.S. est déterminé selon la méthode officielle de Kjeldahl avec un appareil Tecator 
Kjeltec I. La détermination des principales fractions azotées est réalisée par précipitations sélectives suivant la méthode de RowLAND modifiée par AschafFENBURg et DREWRY (1958). Le taux d'azote protéique du lactosérum non dénaturé dans le lait en poudre non gras (Whey Protein Nitrogen Index : W.P.N.I.) est déterminé suivant la méthode citée par HaugaARd Sorensen et al. (1978) et la caséine kappa suivant AlaIs (1985). L'urée est évaluée par la méhode à la diacétylmonoxime (Urée/OCT-KIT coloration 61479 BioMérieux).

\section{Résultats}

\section{A. Stabilité thermique des laits}

Les laits sont reconstitués au laboratoire puis stérilisés en usine à $118^{\circ} \mathrm{C}$ pendant $13 \mathrm{~min}$. L'aspect des lait stérilisés en présence ou non de phosphate disodique est indiqué dans le tableau I.

\section{TABLEAU 1}

Aspect des laits reconstitués après stérilisation. + : lait homogène ; \pm : lait piqué (petits agglomérats) ; - : lait coagulé (coagulation d'une partie importante des protéines) ; - - lait fortement coagulé (coagulation totale avec séparation de sérum)

Reconstituted milks aspect after sterilization. + : homogeneous milk; \pm : quilted milk (little agglomerates); - : coagulated milk (clotting of an important part of proteins); - - very coagulated milk (total clotting with whey separation)

\begin{tabular}{|c|c|c|c|c|c|c|}
\hline \multicolumn{2}{|c|}{ Ajouts de $\mathrm{Na}_{2} \mathrm{HPO}_{4}(\mathrm{mg} / \mathrm{kg})$} & 0 & 300 & 600 & 900 & 1200 \\
\hline Echantillons & $\begin{array}{l}\text { H.H.1. } \\
\text { H.H.2 } \\
\text { M.H.3 } \\
\text { M.H.4 }\end{array}$ & $\begin{array}{l}+ \\
+ \\
--\end{array}$ & $\begin{array}{l}+ \\
+ \\
\pm \\
+\end{array}$ & $\frac{+}{\frac{ \pm}{ \pm}}$ & $\frac{+}{ \pm}$ & $\frac{+}{--}$ \\
\hline
\end{tabular}

\section{B. Analyse des échantillons}

Le classement des poudres en " High Heat » et en " Medium Heat » est déterminé en fonction du W.P.N.I. Les poudres ayant un W.P.N.I. inférieur à 1,50 entrent dans la catégorie H.H. alors qu'un W.P.N.I. compris entre 1,51 et 5,99 correspond à la catégorie M.H. Les W.P.N.I. trouvés pour les poudres H.H.1 et H.H.2 sont respec- 
tivement 1,10 et 1,16 . Les poudres M.H.3 et M.H.4 ont un W.P.N.I. de 2,45 et 3,02 . Il est important de remarquer la similitude des valeurs trouvées pour les échantillons H.H.1 et H.H.2 ainsi que l'indice élevé de M.H.3 qui classe indiscutablement cette poudre dans la catégorie M.H.

La concentration en azote caséinique des laits H.H. est supérieure à celle des laits M.H. Par contre le lait H.H.1 se caractérise par sa faible teneur en caséine $\chi$. Les teneurs en azote soluble et en particulier en $\beta$-lactoglobuline et en $\alpha$-lactalbumine sont nettement plus élevées pour les laits M.H. Ces résultats sont en accord avec les valeurs du W.P.N.I. trouvées pour les 4 échantillons. Les poudres H.H. et M.H. se différencient aussi par leurs teneurs en azote non protéique et en urée (tab. 2).

TABLEAU 2

Détermination des différentes fractions azotées du lait reconstitué à $17,6 \%$ M.S. (gN/100 g).

W.P.N.I. en milligrammes d'azote protéique par gramme de poudre

Determination of nitrogenous compounds contents of reconstituted milk at $17.6 \%$ T.S. (gN/100 g).

W.P.N.I. in milligrams of protein nitrogen per gram of powder

\begin{tabular}{l|c|c|c|c}
\hline \multicolumn{1}{c|}{ Echantilon } & H.H.1 & H.H.2 & M.H.3 & M.H.4 \\
\cline { 2 - 3 } Azote total & 1,028 & 1,024 & 1,056 & 1,058 \\
Caséine & 0,934 & 0,934 & 0,882 & 0,908 \\
Caséine $x$ & 0,115 & 0,153 & 0,165 & 0,159 \\
& 0,094 & 0,090 & 0,174 & 0,150 \\
Azote du lactosérum & 0,012 & 0,008 & 0,051 & 0,033 \\
$\beta$-lactoglobuline & 0,005 & 0,009 & 0,035 & 0,026 \\
a-lactalbumine & 0,027 & 0,024 & 0,026 & 0,032 \\
Globulines & 0,006 & 0,003 & 0,007 & 0,008 \\
Protéose peptone & 0,044 & 0,045 & 0,056 & 0,051 \\
& 0,019 & 0,015 & 0,027 & 0,025 \\
Azote non protéique & 1,10 & 1,16 & 2,45 & 3,02 \\
Urée & & & & \\
W.P.N.I. & & &
\end{tabular}

La teneur en cendres des laits H.H. est légèrement inférieure à celle des laits M.H. Les concentrations des principaux éléments minéraux sont rassemblées dans le tableau 3. 


\section{TABLEAU 3}

Teneurs en éléments minéraux exprimées en milligrammes pour 100 grammes de lait reconstitué à $17,6 \%$ M.S.

Inorganic compounds contents expressed in milligrams for 100 grams of reconstituted milk at $17.6 \%$ T.S.

\begin{tabular}{|c|c|c|c|c|}
\hline Echantillon & H.H.1 & Н.Н.2. & M.H.3 & M.H.4 \\
\hline $\begin{array}{l}\text { Cendres } \\
\text { Calcium } \\
\text { Calcium soluble } \\
\text { Calcium ionique } \\
\text { Magnésium } \\
\text { Magnésium soluble } \\
\text { Potassium } \\
\text { Sodium } \\
\text { Phosphore } \\
\text { Phosphore soluble }\end{array}$ & $\begin{array}{r}1460 \\
235 \\
48 \\
14,1 \\
29 \\
9 \\
331 \\
98 \\
189 \\
48\end{array}$ & $\begin{array}{c}1440 \\
244 \\
52 \\
13,5 \\
23 \\
9 \\
321 \\
85 \\
179 \\
51\end{array}$ & $\begin{array}{c}1500 \\
242 \\
53 \\
13,9 \\
29 \\
11 \\
348 \\
83 \\
182 \\
57\end{array}$ & $\begin{array}{r}1510 \\
245 \\
52 \\
14,2 \\
25 \\
10 \\
319 \\
86 \\
188 \\
54\end{array}$ \\
\hline
\end{tabular}

C. Effet du $p H$

Le tracé des courbes du temps de coagulation en fonction du $\mathrm{pH}$ (courbes T.C.-pH) est particulièrement intéressant pour caractériser le comportement thermique des échantillons dont les $\mathrm{pH}$ naturels sont très proches et les T.C. correspondants du même ordre de grandeur, excepté pour l'échantillon M.H.4 qui est moins stable (fig. 2). Les échantillons sont totalement déstabilisés lorsque le $\mathrm{pH}$ est supérieur de 0,1 unité au $\mathrm{pH}$ naturel et aucune remontée du T.C. n'est observée entre les $\mathrm{pH}$ 6,70 et 7,50. Le lait H.H.1 a une bonne stabilité aux pH compris entre 6,52 et 6,60 et la courbe présente une symétrie par rapport au $\mathrm{pH}$ de stabilité maximale. Cette symétrie ne se retrouve pas pour l'échantillon H.H.2 puisqu'une élévation de $\mathrm{pH}$ de 0,03 unité se traduit par une chute rapide du T.C. et que son acidification s'accompagne d'une diminution modérée du T.C. Le lait M.H.3 présente une stabilité importante entre les pH 6,48 et 6,58 et une déstabilisation brutale lorsque le $\mathrm{pH}$ s'écarte de cet intervalle. L'échantillon M.H.4 a une stabilité thermique nettement inférieure aux 3 autres poudres et la déstabilisation intervient rapidement dès que le $\mathrm{pH}$ est modifié. Ces résultats sont compatibles avec les essais de stérilisation industrielle effectués aux $\mathrm{pH}$ 6,456,52-6,65. Ces mêmes essais montrent que l'abaissement du pH pendant la stérilisation est d'autant plus faible que le $\mathrm{pH}$ initial est bas. 


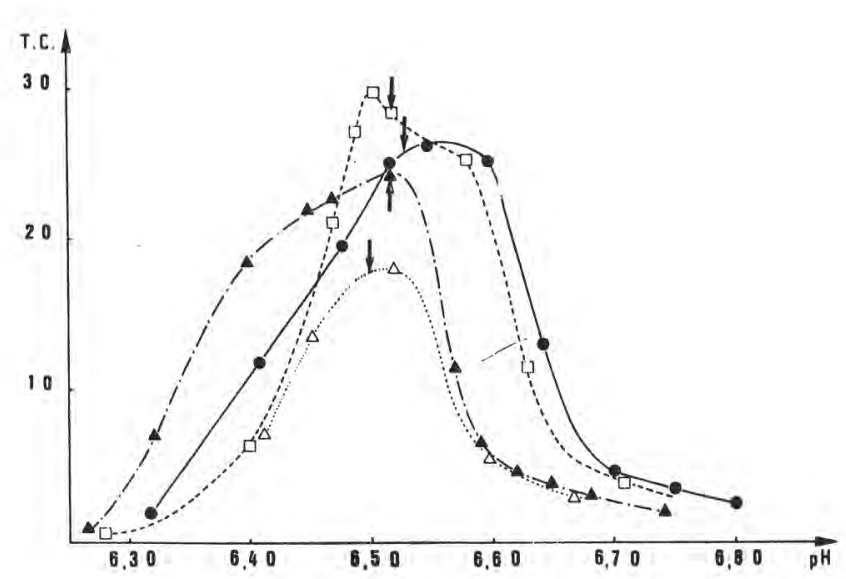

FIG. 2

Variations du temps de coagulation (T.C.) des laits reconstitués à $17,6 \%$ d'extrait sec en fonction du pH. • : lait H.H.1; $\mathbf{\Delta}$ : lait H.H.2; $\mathrm{\text {: }}$ : lait M.H.3; $\triangle$ : lait M.H.4 ; $\rightarrow$ : $p H$ naturel.

Coagulation time (T.C.) variations of reconstituted milks at $17,6 \%$ dry extract as a function of pH. $\bullet$ : milk H.H.I ; $\mathbf{\Delta}$ milk H.H.2 ; 口 milk M.H.3; $\triangle$ : milk M.H.4 ; $\rightarrow$ natural $\mathrm{pH}$.

\section{Effet du calcium}

Le calcium est ajouté sous forme de $\mathrm{CaCl}_{2}$ au lait reconstitué à $17,6 \%$ M.S. Les variations de la force ionique dues aux différences entre les échantillons sont compensées par une addition simultanée et complémentaire de $\mathrm{KCl}$ de sorte que l'augmentation totale de la force ionique soit constamment égale à 0,006 ou à 0,015 moles par litre.

L'ajout de calcium provoque de façon globale une diminution modérée de la thermostabilité des laits. Toutefois chaque poudre a un comportement qui lui est propre (fig. 3). Le T.C. du lait H.H.1 à pH 6,65 est sensiblement augmenté en présence d'un ajout de calcium inférieur à $12 \mathrm{mg} / 100 \mathrm{~g}$ de lait. Pour les laits M.H., une légère amélioration est notée par rapport au témoin aux $\mathrm{pH}$ inférieurs à 6,58 après une addition de $4 \mathrm{mg} \mathrm{Ca} / 100 \mathrm{~g}$; par contre si la force ionique est augmentée de 0,006 moles par litre au lieu de 0,015 moles par litre, cette amélioration disparaît et le T.C. est d'autant plus faible que la concentration en calcium est élevée.

A la suite d'un ajout de $20 \mathrm{mg} \mathrm{Ca} / 100 \mathrm{~g}$ de lait, 78 à $82 \%$ du calcium se trouvent sous forme insoluble; simultanément une chute du phosphore soluble est observée. Après stérilisation, les teneurs en calcium soluble et en phosphore soluble diminuent quand la quantité de $\mathrm{CaCl}_{2}$ ajoutée s'élève. 

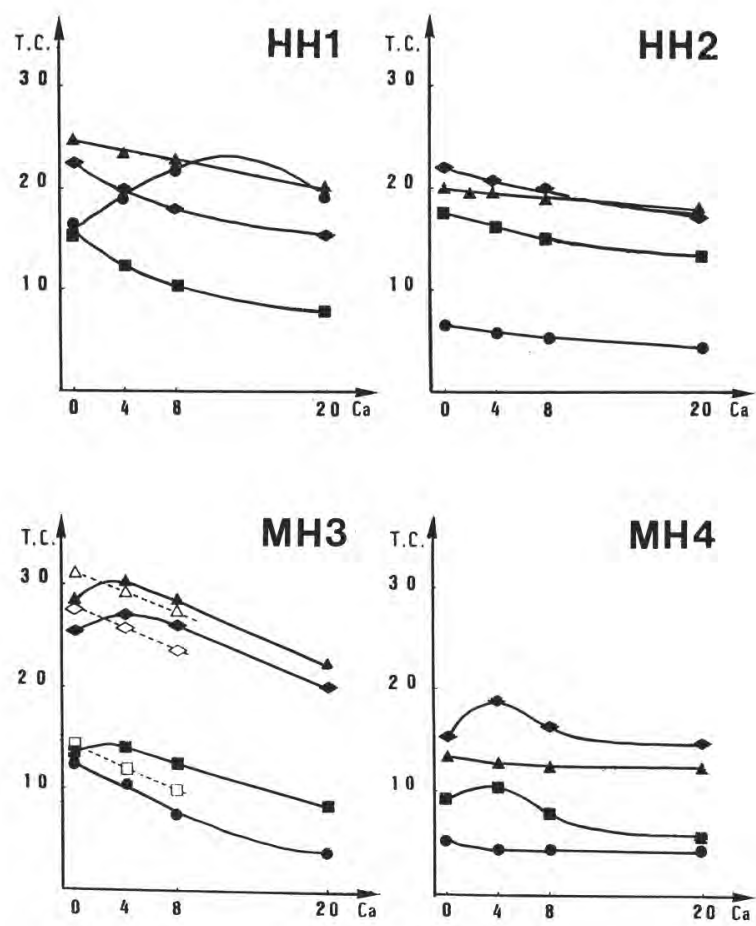

FIG. 3

Evolution du temps de coagulation (T.C.) en fonction du pH et du calcium ajouté à $100 \mathrm{~g}$ de lait reconstitué ( $\mathrm{mg} \mathrm{Ca} / 100 \mathrm{~g}$ ).

Augmentation de la force ionique de 0,006 mol. $/ \mathrm{l}$.

Augmentation de la force ionique de $0,015 \mathrm{~mol} . / \mathrm{l}$.

ㅁ: $p H 6,45 ; \diamond: p H 6,52 ; \triangle: p H 6,58$.

- $p H 6,45 ;: p H 6,52 ; \mathbf{\Delta}: p H 6,58$;

- : pH 6,65.

Evolution of coagulation time (T.C.) as a function of $\mathrm{pH}$ and added calcium in

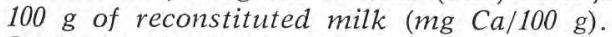

Increase of ionic strengh of $0.006 \mathrm{~mol} . / \mathrm{l}$.

Increase of ionic strengh of $0.015 \mathrm{~mol} . / \mathrm{l}$.

口: $p H 6.45 ; \infty: p H 6.52 ; \triangle: p H 6.58$.

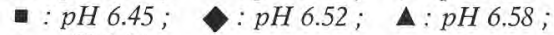

- : $p H 6.65$.

\section{E. Effet du phosphate disodique}

L'ajout de phosphate disodique au lait reconstitué aboutit à une augmentation du T.C. au pH 6,45 et à une diminution du T.C. au pH 6,65 (fig. 4). Aux pH intermédiaires, les variations du T.C. sont liées aux types de poudre ; avec les échantillons H.H., l'amélioration de la stabilité thermique grâce au phosphate est peu accentuée et limitée aux pH 6,45 et 6,52. L'effet du phosphate sur les laits M.H. se manifeste sur une plage de $\mathrm{pH}$ plus large et provoque une nette augmentation du T.C. Le déplacement du maximum de stabilité vers les $\mathrm{pH}$ acides à la suite de l'addition de phosphate est plus marqué 


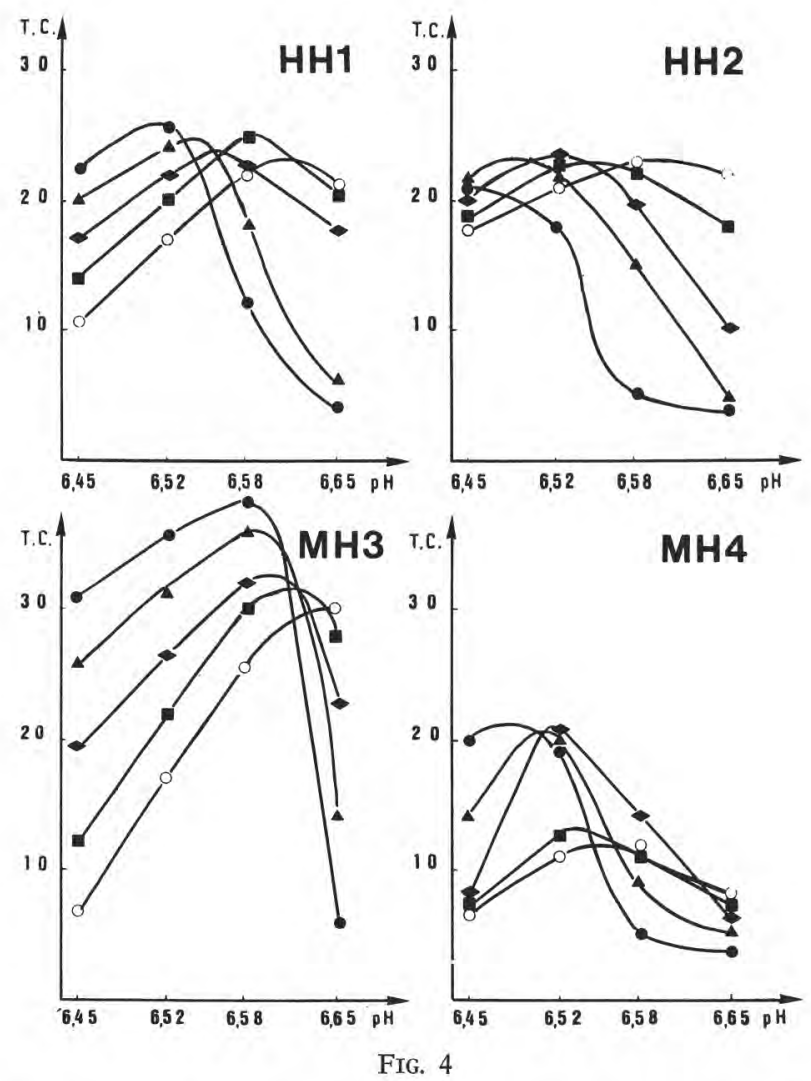

Courbes des temps de coagulation (T.C.) en fonction $d u p H$ du lait et de l'addition de phosphate disodique $(m \mathrm{~m} P / 100 \mathrm{~g})$. 0 : témoin ; : $+2,18$; : $+6,55 ; \mathbf{\Delta}:+13,1 ; \bullet:+19,6$.

Coagulation time (T.C.) curves as a function of milk $p H$ and added di-sodium phosphate (mg P/100 g). 0 : check sample; : $+2.18 ;-+6,55 ; \mathbf{\Delta}: 13.1$; - +19.6 .

pour les échantillons H.H. (0,10 unité de $\mathrm{pH})$ que pour les échantillons M.H. (0,05 unité de $\mathrm{pH})$.

Le phosphore ajouté au lait reconstitué reste essentiellement sous forme soluble (H.H.1 $85 \%$, H.H.2 $77 \%$, M.H.3 77 \%, M.H.4 $100 \%$ ) et on ne constate qu'une insolubilisation négligeable du calcium. Après stérilisation du lait à $\mathrm{pH} 6,52$, la teneur en phosphore soluble augmente linéairement en fonction de la quantité de phosphate ajoutée alors que la concentration en calcium soluble diminue ; cette évolution est indépendante du T.C. Les rapports molaires du calcium insolubilisé ou phosphore insolubilisé après stérilisation en présence d'un ajout de $21,8 \mathrm{mg}$ de phosphore dans $100 \mathrm{~g}$ de lait aboutissent à des écarts minimes entre les laits H.H.1 et H.H.2 qui 
se distinguent nettement des valeurs trouvées pour M.H.3 tant au pH 6,52 que 6,65 (tabl. 4).

\section{TABLEAU 4}

Rapports moléculaires du calcium insolubilisé au phosphore insolubilisé au cours de la stérilisation d'un lait renfermant un ajout de $21,8 \mathrm{mg} P / 100 \mathrm{~g}$

Molecular ratios of insolubilised calcium on insolubilised phosphorus during the sterilization of a milk with an addition of $21.8 \mathrm{mg} P / 100 \mathrm{~g}$

\begin{tabular}{rccc}
\hline Echantillon & H.H.1 & H.H.2 & M.H.3 \\
\hline pH 6,52 & 0,39 & 0,39 & 0,50 \\
pH 6,65 & 0,38 & 0,35 & 0,23 \\
\hline
\end{tabular}

\section{F. Effet de la force ionique}

L'influence de la force ionique sur la stabilité thermique des laits reconstitués est déterminée par additions de $\mathrm{KCl}$ jusqu'à une augmentation de 0,038 moles par litre, soit environ 1,24 fois la force ionique d'un lait reconstitué à $17,6 \%$ M.S. Cette élévation est équivalente à celle due à l'addition de $90 \mathrm{mg}$ de $\mathrm{Na}_{2} \mathrm{HPO}_{4}$ à $100 \mathrm{~g}$ de lait. Une telle addition de $\mathrm{KCl}$ n'intervient pas sur le $\mathrm{pH}$ ni sur le phosphore soluble ou le calcium soluble du lait reconstitué. Après stérilisation des laits dont le $\mathrm{pH}$ a été préalablement ajusté à 6,52 ou 6,65, une baisse significative du phosphore soluble et du calcium soluble est observée pour les laits H.H.1, H.H.2, et M.H.3.

Les courbes T.C.-pH (fig. 5) montrent l'effet déstabilisant suscité par l'accroissement de la force ionique aux $\mathrm{pH}$ inférieurs ou égaux aux $\mathrm{pH}$ naturels des laits reconstitués. Quand le $\mathrm{pH}$ des échantillons est augmenté, une élévation du T.C. est esquissée au $\mathrm{pH}$ 6,58 puis confirmée au pH 6,65.

\section{G. Effet de l'urée}

L'addition de quantités d'urée allant jusqu'à $100 \mathrm{mg}$ pour $100 \mathrm{~g}$ de lait n'amène pas de changement notable de $\mathrm{pH}$ ni d'effet tampon; le tracé de la courbe de titration entre le $\mathrm{pH} 5,0$ et le $\mathrm{pH}$ 9,5 d'un lait auquel $100 \mathrm{mg}$ d'urée sont ajoutés se superpose exactement avec celui du lait témoin sans ajout d'urée. Les dosages du phosphore soluble et du calcium soluble sur le lait à $\mathrm{pH}$ 6,52 avant et après stérilisation ne montrent pas d'évolution de ces constituants quand la concentration en urée augmente. 

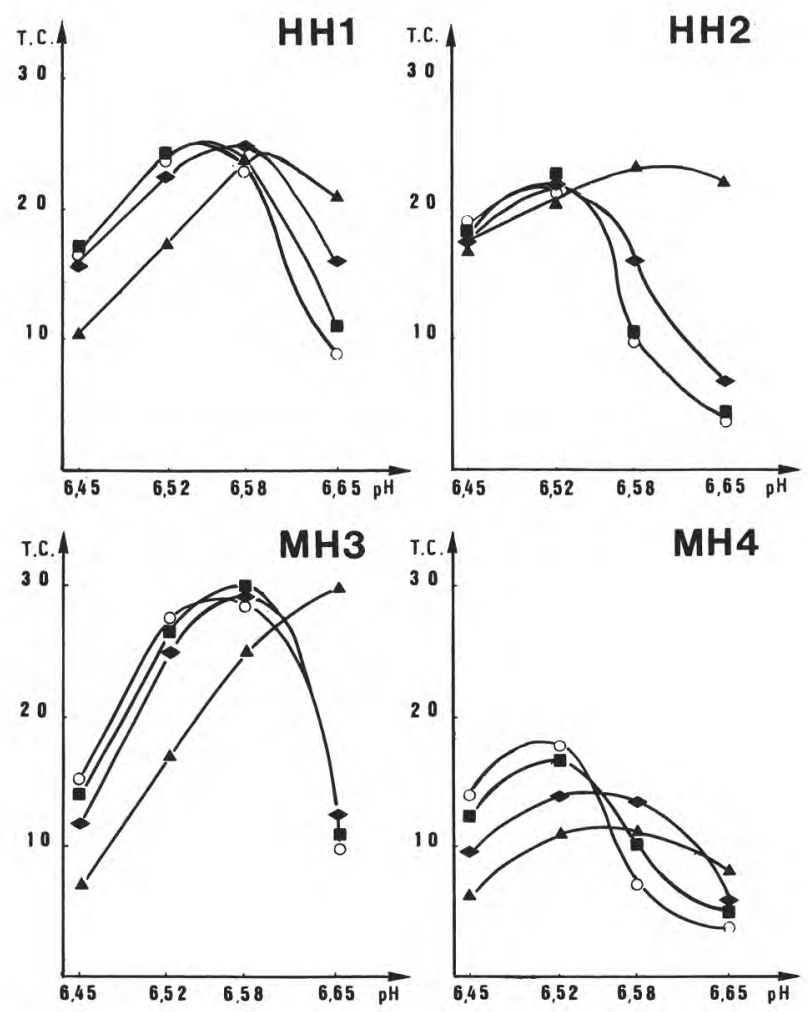

FIG. 5

Courbes des temps de coagulation (T.C.) en fonction du pH et de l'augmentation de la force ionique du lait. $\mathrm{O}$ : témoin ; : $+0,006 \mu ;$ A . $+0,038 \mu$.

Curves of coagulation time (T.C.) as a function of $\mathrm{pH}$ and ionic strengh increase of milk. O : check sample; : $+0.006 \mu ; \quad+0,015 \mu ; \mathbf{\Lambda}:+0.038 \mu$.

L'augmentation linéaire du T.C. d'un lait à pH 6,52 est limitée à de faibles additions d'urée. Lorsque la teneur en urée croît, le T.C. passe par un maximum puis devient rapidement inférieur à celui du témoin (fig. 6). L'augmentation de la teneur en urée se traduit par un déplacement du T.C. maximum vers les $\mathrm{pH}$ acides.

\section{Discussion et conclusion}

La technique de reconstitution des poudres de lait écrémé suivie de la mesure du T.C. à $130^{\circ} \mathrm{C}$ donne des résultats reproductibles. 

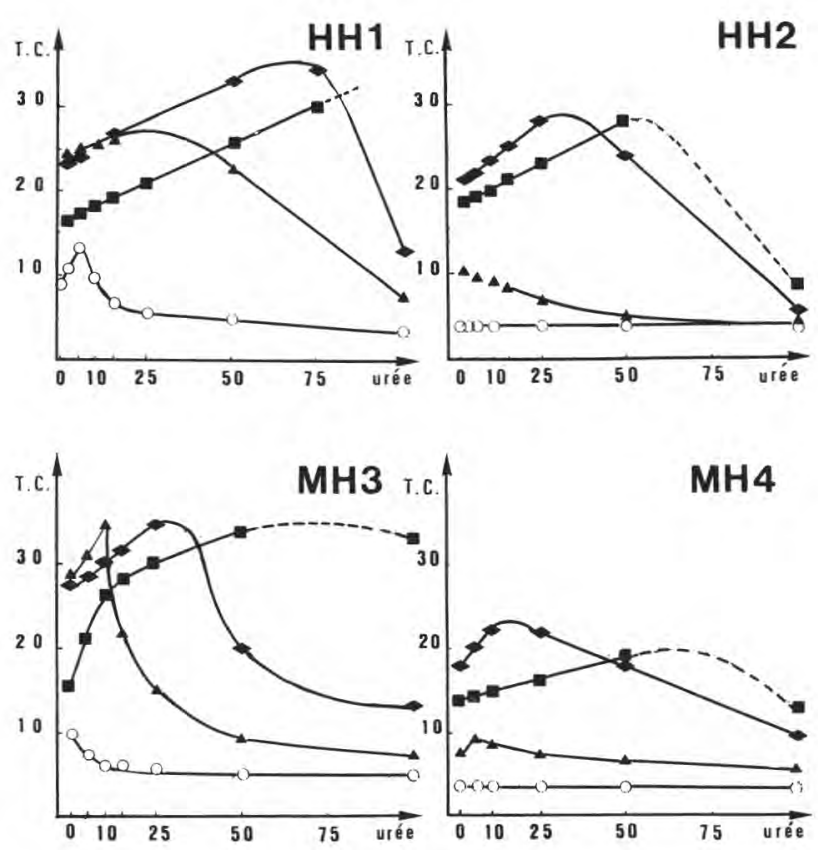

FIG. 6

Variations du temps de coagulation (T.C.) en fonction du pH et de l'urée ajoutée $(m g / 100 \mathrm{~g})$. : $p H \quad 6,45 ;: p H \quad 6,52 ; \mathbf{\Delta} p H 6,58 ; \circ p H 6,65$.

Coagulation time (T.C.) variations as a function of $\mathrm{pH}$ and of added urea $(m g / 100 \mathrm{~g})$. : $p H 6.45 ; \diamond: p H 6.52 ; \boldsymbol{\Lambda}: p H 6.58 ; 0: p H 6.65$.

Bien que les conditions de mise en solution des poudres et les traitements thermiques des laits au laboratoire soient sensiblement différents de ceux utilisés dans l'industrie, les valeurs obtenues lors des mesures du T.C. ne sont pas en contradiction avec l'aspect des laits après une stérilisation industrielle.

La comparaison des teneurs en composés azotés et en éléments minéraux des 4 échantillons (voir tabl. 2 et 3) ne permet pas d'infirmer ou de confirmer les données bibliographiques se rapportant à l'influence de certains constituants analysés sur la stabilité thermique des laits (Fox et NASH, 1979 ; Holt et al., 1978 b ; Rose, 1961 ; SWeEtsur et White, 1974 ; SWeetsur et White, 1975). Cependant plusieurs observations peuvent être faites. La concentration en azote total et en cendres des poudres H.H. est inférieure à celle des laits M.H. Cette différence de l'ordre de $3 \%$ peut être expliquée par un préchauffage plus sévère appliqué lors de la préparation des poudres H.H. aboutissant à des pertes sensibles de protéines du lactosérum par dépôt sur les parois des échangeurs thermiques. 
La détermination des différentes fractions des protéines du lait effectuée sur les laits reconstitués (voir tabl. 2) confirme l'influence du mode de préparation des poudres sur les protéines solubles; les teneurs en protéines du lactosérum non dénaturées ( $\beta$-lactoglobuline et o-lactalbumine) des laits H.H. sont nettement inférieures à celles des laits M.H. La concentration en caséine des laits H.H. semble supérieure à celle trouvée pour les laits M.H., en raison de la combinaison plus importante des protéines du sérum avec la caséine au cours du chauffage. Si la formation du complexe caséine- $\beta$-lactoglobuline améliore généralement la stabilité thermique du lait, ce processus n'est pas suffisant pour déterminer l'aptitude d'un lait à subir une stérilisation.

Les échantillons H.H. étudiés se caractérisent par des W.P.N.I., c'est-à-dire des teneurs en protéines du sérum non dénaturées, très proches; toutefois leur comportement au cours d'une stérilisation présente peu de similitudes : le lait H.H.1 est stable en présence ou non de phosphate disodique alors que le lait H.H.2 coagule si l'ajout de phosphate n'est pas optimum. De même les échantillons M.H. ont un W.P.N.I. élevé qui les classe parmi les laits incompatibles avec un traitement de stérilisation alors que le lait reconstitué M.H.3 présente un T.C. supérieur à celui des laits H.H. étudiés. Il apparaît que les différentes fractions protéiques des laits reconstitués ne présentent pas de relation étroite avec le T.C. Seule la teneur naturelle en urée est liée avec le T.C. des laits H.H.1, H.H.2, M.H.3. Cette constatation rejoint les résultats de MuIR et SWEETSur (1976) qui avaient établi une relation entre le temps de coagulation et la teneur en urée de laits de grand mélange.

La disparité des résultats se rapportant aux éléments minéraux n'autorise pas l'établissement de liens entre ces concentrations et les temps de coagulation des laits. Les données bibliographiques contradictoires se rapportant à la relation existant entre la composition du lait et sa stabilité thermique (Fox, 1982; Holt et al., 1978; SWeETSUR et WhITE, 1975) soutiennent ce point de vue. Il est vraisemblable que le calcium et le phosphore interviennent indirectement sur le T.C. en favorisant certaines réactions comme la diminution de la répulsion intermoléculaire à la suite de la fixation de calcium sur les groupements ionisés des protéines ou la modification de l'équilibre phosphocalcique de la caséine. Par conséquent, ce ne sont peut-être pas les concentrations qui sont déterminantes par elles-mêmes mais la nature des réactions que ces éléments sont susceptibles de réaliser en fonction des conditions du milieu.

SWEetsur et White (1975) montrent que l'augmentation de la teneur en protéines lors de la concentration du lait et l'élévation de l'acidité au cours du chauffage sont les 2 principaux facteurs contribuant à la diminution de la stabilité thermique des laits concentrés. Les courbes T.C.-pH établies à $130^{\circ} \mathrm{C}$ à partir de laits concentrés reconstitués (voir fig. 2) font apparaître une seule zone 
de stabilité réduite à un intervalle de $\mathrm{pH}$ beaucoup plus étroit $(0,2$ unité de $\mathrm{pH})$ que pour les laits normaux.

Chaque échantillon étudié est caractérisé par sa courbe T.C.-pH alors que les $\mathrm{pH}$ naturels et les $\mathrm{pH}$ du maximum de stabilité des différents laits sont pratiquement identiques. Le réajustement du $\mathrm{pH}$ du lait reconstitué avant la stérilisation n'apporte pas d'amélioration sensible du T.C.; par contre la position du $\mathrm{pH}$ naturel sur la courbe T.C.-pH peut se révéler plus intéressante. L'échantillon H.H.2 se distingue par une chute rapide du T.C. dès que le $\mathrm{pH}$ devient supérieur au pH naturel alors que les autres échantillons voient leur T.C. varier plus légèrement lorsque le $\mathrm{pH}$ est augmenté de 0,05 unité. Cette différence laisse présager une faible stabilité du lait H.H.2 à la stérilisation malgré son W.P.N.I. correct. D'autre part, aux pH inférieurs au pH naturel, les courbes T.C.-pH ont un profil différent pour chaque lait, par contre aux $\mathrm{pH}$ supérieurs au $\mathrm{pH}$ naturel la pente est identique pour les 4 échantillons $(2,8 \mathrm{~min}$ pour une augmentation de 0,01 unité de $\mathrm{pH}$ ). Le mécanisme de déstabilisation apparaît moins dépendant de la nature de l'échantillon aux $\mathrm{pH}$ élevés qu'aux $\mathrm{pH}$ inférieurs au $\mathrm{pH}$ naturel. Ce phénomène semblerait indiquer un processus de déstabilisation différent de part et d'autre du pH naturel.

L'addition de calcium n'entraîne pas systématiquement un abaissement du T.C. Les courbes T.C. en fonction de la quantité de calcium ajoutée établies pour différents $\mathrm{pH}$ (fig. 3) laissent paraître une déstabilisation aux $\mathrm{pH}$ inférieurs à 6,58 ; ce phénomène est peu ou pas observé aux pH plus élevés, probablement en raison de la diminution de la solubilité du calcium quand le $\mathrm{pH}$ augmente. Les échantillons H.H. et en particulier H.H.1 se différencient des laits M.H. par un déplacement sensible du maximum de stabilité vers les $\mathrm{pH}$ élevés. Après une addition de $4 \mathrm{mg} \mathrm{Ca} / 100 \mathrm{~g}$ de lait, une faible augmentation du T.C. par rapport au témoin est observée pour les échantillons M.H. aux pH 6,45 et 6,52. La même expérience réalisée après avoir ajusté l'élévation de la force ionique à $+0,006$ au lieu de $+0,015$ moles par litre n'entraîne pas cette amélioration. La différence de comportement des laits M.H. ainsi mise en évidence peut être due soit à la valeur de la force ionique, soit à la teneur en potassium particulièrement élevée pour le témoin à $+0,015$ moles par litre; la première hypothèse est la plus probable.

Les teneurs en calcium soluble et en phosphore soluble déterminées après addition de $\mathrm{CaCl}_{2}$ au lait non stérilisé, de même que leur rapport molaire, ne permettent pas de définir sous quelles formes se retrouvent le calcium et le phosphore insolubilisés. Pour cela, il conviendrait d'établir si ces éléments insolubilisés se retrouvent associés à la structure des micelles ou simplement sous forme de composés insolubles; une chromatographie par perméation de gel sur colonne pourrait apporter des indications sur la destinée du calcium et du phosphore à la suite de l'addition progressive de 
$\mathrm{CaCl}_{2}$. Il apparaît d'une part que les teneurs en calcium total, en calcium soluble et en calcium ionique des laits concentrés reconstitués ne sont pas liées au T.C. à $130^{\circ} \mathrm{C}$ et d'autre part que l'ad.dition de $\mathrm{CaCl}_{2}$ n'est responsable que d'une déstabilisation modérée aux $\mathrm{pH}$ inférieurs au $\mathrm{pH}$ naturel. Ces 2 observations laissent supposer que le calcium n'est vraisemblablement pas un facteur déterminant du processus de déstabilisation thermique du lait.

L'addition de quantités croissantes de $\mathrm{Na}_{2} \mathrm{HPO}_{4}$ provoque un déplacement du maximum de stabilité vers les $\mathrm{pH}$ acides d'autant plus faible que l'augmentation du T.C. maximum est importante (voir fig. 4). Les échantillons H.H. ont leur T.C. maximum peu modifié alors que les laits M.H. voient leur T.C. maximum fortement accru. Autrement dit, si l'effet stabilisant du préchauffage est important, le phosphate disodique aura peu d'effet et réciproquement. Cela voudrait dire :

- que les effets du préchauffage et du phosphate sont de même nature et mettent en jeu le même phénomène;

- qu'ils ne sont cumulables que si le phénomène en question n'a pas été réalisé en totalité.

Comme Muller (1965), nous constatons que l'efficacité du phosphate paraît liée au W.P.N.I. de la poudre d'origine. De plus, il est important de remarquer que le profil de la courbe T.C.-pH des laits concentrés reconstitués non modifiés est d'autant plus accentué entre les $\mathrm{pH}$ 6,40 et 6,50 que l'effet du $\mathrm{Na}_{2} \mathrm{HPO}_{4}$ sur le T.C. maximum est important. Cette relation expliquerait en partie que l'acidité et le pH d'un lait ne permettent pas de déterminer la quantité de phosphate à rajouter pour obtenir un produit stable au cours de la stérilisation. L'amélioration de la stabilité thermique aux $\mathrm{pH}$ inférieurs au $\mathrm{pH}$ optimum du témoin ne doit pas s'expliquer uniquement par la réaction du phosphate sur le calcium ionique dont la concentration est d'autant plus élevée que le $\mathrm{pH}$ est bas. Un tel processus confèrerait au calcium un rôle prépondérant dans le mécanisme de coagulation thermique du lait, ce qui va à l'encontre des conclusions tirées de notre étude sur l'effet du calcium sur la thermostabilité. D'autre part, l'efficacité du $\mathrm{Na}_{2} \mathrm{HPO}_{4}$ varie suivant que la poudre utilisée est du type H.H. ou M.H. et cela malgré des teneurs en calcium total, en calcium soluble et en calcium ionique similaires pour les 4 laits étudiés après reconstitution.

La teneur en phosphore soluble des laits stérilisés après ajustement du $\mathrm{pH}$ à 6,52 augmente linéairement avec la quantité de phosphate ajoutée; simultanément les teneurs en calcium soluble et en calcium ionique diminuent aussi de façon linéaire. Ces variations sont indépendantes du T.C. et de la stabilité des laits au cours d'une stérilisation en usine. L'action du phosphate ne se limite donc pas à son pouvoir chélateur. Selon Horne (1982) le phosphate, à la différence de l'acide citrique, intervient dans le mécanisme de précipitation de la caséine $\alpha_{s_{1}}$ en plus de son pouvoir chélateur du cal- 
cium. Cette constatation n'explique pas la relation existant entre le W.P.N.I. et l'efficacité du phosphate. La mise en évidence des composés organiques réagissant avec les phosphates au cours de la stérilisation constitue certainement une étape indispensable pour définir le rôle du phosphate sur la thermostabilité des laits.

L'addition de $\mathrm{CaCl}_{2}$ ou de $\mathrm{Na}_{2} \mathrm{HPO}_{4}$ aboutit à des variations de stabilité thermique qui peuvent être attribuées à l'activité propre des ions ajoutés mais aussi à l'élévation de la force ionique des laits étudiés. Les variations de la force ionique liées à l'addition de quantités croissantes de $\mathrm{CaCl}_{2}$ et de $\mathrm{Na}_{2} \mathrm{HPO}_{4}$ sont théoriquement uniformisées par l'addition de $\mathrm{KCl}$. En fait, cet ajustement ne prend pas en considération l'insolubilisation d'une partie du calcium ou du phosphore ajouté. Par conséquent, il est difficile d'apprécier dans quelle mesure la force ionique intervient sur la thermostabilité lors de l'addition de $\mathrm{CaCl}_{2}$ ou de $\mathrm{Na}_{2} \mathrm{HPO}_{4}$. Il est donc intéressant de comparer les résultats précédents avec ceux obtenus lorsque l'élévation de la force ionique n'est due qu'à un élément totalement dissocié dans le sérum et n'intervenant pas a priori par lui-même dans les réactions commandant la thermostabilité.

L'influence de la force ionique apparaît clairement sur les courbes T.C.-pH de la figure 5 : le maximum de stabilité est déplacé vers les $\mathrm{pH}$ alcalins et les T.C. enregistrés aux $\mathrm{pH}$ acides diminuent quand la force ionique croît. Ces résultats ne correspondent pas à ceux trouvés par Fox et NASH (1979) et SwEETSUR et WHITE (1975) à partir du lait normal dont la force ionique est élevée soit par addition de $\mathrm{NaCl}$ soit par augmentation des sels minéraux par dialyse. Dans le premier cas, l'ajout de $\mathrm{NaCl}$ jusqu'à $100 \mathrm{mM} / 1$ ne provoque pas de modification de la stabilité thermique si le $\mathrm{pH}$ du lait est inférieur ou égal au $\mathrm{pH}$ du maximum de stabilité et dans le $2^{\mathrm{e}}$ cas une augmentation du T.C. est observée dans la même gamme de $\mathrm{pH}$.

Il est actuellement bien établi que la stabilité thermique d'un lait est liée à la teneur en urée (MUIR et SweEtSuR, 1976 ; MuIr et SWEESUR, 1977 ; Holt et al., 1978 a). L'addition d'urée au moment de 1a reconstitution d'un lait concentré aboutit à une amélioration de la thermostabilité (KELLY, 1977) à condition que la poudre soit issue d'un lait récolté en milieu de saison (KELLY, 1982). La relation entre la concentration en urée et le T.C. est retrouvée pour les laits H.H. et M.H. étudiés mais uniquement pour de faibles ajouts.

Le tracé de la courbe T.C. en fonction de la quantité d'urée ajoutée montre qu'au delà d'une concentration optimale les temps de coagulation diminuent progressivement jusqu'à des valeurs nettement inférieures à celle du témoin. L'influence bénéfique de l'urée, de même que son effet déstabilisateur lorsque la quantité d'urée ajoutée dépasse la valeur optimale, ne s'accompagne pas de modification du $\mathrm{pH}$, de l'effet tampon, de la force ionique ni de variation de la concentration en phosphore soluble, en calcium soluble ou en calcium ionique. D'autre part, l'effet de l'urée est du même type 
pour les 4 échantillons bien que leur W.P.N.I. soit compris entre 1,10 et 3,02 . Il résulte de ces constatations que le mode d'action de l'urée doit être rechenché au niveau des protéines de la phase colloïdale. Deux hypothèses de travail peuvent être retenues :

- 1'urée agit sur la thermostabilité suivant un mécanisme différent de celui mettant en cause le phosphore, le calcium, le pH, la force ionique ou les protéines du sérum;

- les variations des teneurs en protéines solubles, en phosphore, en calcium ou les modifications du $\mathrm{pH}$ et de la force ionique ne sont qu'une conséquence des transformations du système caséique ou des réactions secondaires induites par le traitement thermique.

Nous avons limité notre étude à des paramètres intéressant la phase soluble. Tous interviennent dans la déstabilisation du système caséinate, soit en modifiant la structure des caséines, soit à la suite de dépôts de composés insolubilisés, organiques ou minéraux, à la surface des micelles dont les caractéristiques sont ainsi modifiées. La maîtrise de la stabilité thermique des laits concentrés reconstitués semble donc passer par la détermination qualitative et quantitative des processus responsables de ces 2 types d'altérations des micelles.

\section{Remerciements}

Nous remercions la Société Industrielle de Produits Laitiers (S.I.P.L.) de Dakar où ont été réalisés les essais industriels.

\section{Summary}

\section{Studies on the heat stability of reconstituted concentrated milks}

The heat stability study of reconstituted concentrated milks was performed on skim milk powders of « High Heat» (H.H.) and « Medium Heat " (M.H.) type. The heat stability was estimated by coagulation time measurement at the laboratory and by a visual inspection after industrial sterilization. The coagulation time measurements of reconstituted milks were in accordance with results observed in industrial sterilization.

The comparison of nitrogenous and inorganic compound contents of four samples, together with the Whey Protein Nitrogen Index determination (W.P.N.I.) did not allow to determine the milk ability to undergo a sterilization. Only the natural concentration of urea was correlated to coagulation time of milk.

Each sample studied was characterized by its coagulation time vs. $\mathrm{pH}$ curve, when natural $\mathrm{pH}$ and maximum stability $\mathrm{pH}$ of different milks were virtually similar. At $\mathrm{pH}$ lower than natural, the coagulation time vs. $\mathrm{pH}$ curves had a different shape for each milk, whereas at higher $\mathrm{pH}$, the shape was the same for the four samples. This phenomenon could 
indicate a different destabilization process on both sides of the natural $\mathrm{pH}$.

The addition of $\mathrm{CaCl}_{2}$ during reconstitution only induced a moderate destabilization at $\mathrm{pH}$ below 6.58 and did not bring about noticeable changes of the coagulation time at $\mathrm{pH}$ above 6.58 . On the other hand, it seems that the total calcium, soluble calcium and ionic calcium contents of reconstituted condensed milks were not related to coagulation time.

The addition of increasing quantities of $\mathrm{Na}_{2} \mathrm{HPO}_{4}$ to the H.H. samples modified slightly maximal coagulation times whereas M.H. milks had their maximal coagulation time considerably increased; this leads to think that preheating and phosphate addition bring into the same phenomenon.

If the increase of the ionic strength was obtained by addition of $\mathrm{KCl}$ and not by addition of $\mathrm{CaCl}_{2}$ or $\mathrm{Na}_{2} \mathrm{HPO}_{4}$, the maximum stability occured at higher $\mathrm{pH}$ and coagulation time observed at acid $\mathrm{pH}$ decreased when ionic strengh increased.

Urea addition improved heat stability until an optimal content, depending on the samples, was reached beyond which a considerable loss of stability appeared. Urea addition did not induce any $\mathrm{pH}$ modification, change of the buffer capacity, change of soluble phosphate, soluble calcium or ionic calcium concentrations. On the other hand, the effect of urea addition was similar for all samples although their W.P.N.I. were very different. It results from these considerations that the urea could act on the protein colloïdal phase rather than on the soluble phase.

Key words : Concentrated milk - Reconstituted milk - Heat stability.

\section{Références bibliographiques}

ALAIS C., 1984. Science du lait : principes des techniques laitières. Société d'édition et de publicité agricoles, industrielles et commerciales, Paris.

AschafFENBURg G., DREWRY J., 1959. New procedure for the routine determination of the various non-casein proteins of milk. $15^{e}$ Congrès Int. Laiterie, 5, 16311635.

Darling D.F., 1980. Heat stability of milk. J. Dairy Res., 47, 199-210.

Fox P.F., NASH B.M., Physicochemical characteristics of casein micelles ni dilute aqueous media. J. Dairy Res., 46, 357-363.

Fox P.F., 1982. Developments in dairy chemistry. I. Proteins. Applied Science Publishers, London and New York.

Haugaard S $\varnothing$ rensen I., Krag J., Pisecky J., Westergaard V., 1978. Méthodes d'analyses des produits laitiers déshydratés. 4e édition, Niro Atomizer, 305 Gladsaxevej. DK-2860 Soeborg, Denmark.

Holt C., Mutr D.D., SweEtsur A.W.M., 1978 a. The heat stability of milk and concentrated milk containing added aldehydes and sugars. J. Dairy Res., $45,47-52$.

Holt C., Muir D.D., Sweetsur A.W.M., 1978 b. Seasonal changes in the heat stability of milk from creamery silos in south west Scotland, J. Dairy Res., $45,183-190$.

HORNE D.S., 1982. Calcium induced precipitation of $\alpha_{s_{1}}$-casein : effect of inclusion of citrate or phosphate. J. Dairy Res., 49, 107-118.

JenSEN G.K., Nielsen P., 1982. Reviews of the progress of Dairy Science : Milk powder and recombination of milk and milk products. J. Dairy Res., 49, 515-544. 
KELLY P.M., 1977. Effect of some non-protein nitrogen components on the heat stability of skim milk powders. Ir. J. Food Sci. Technol., 1, 129-135.

KelLy P.M., 1982. The effect of preheat temperature and urea addition on the seasonal variation in the heat stability of skim milk powder. J. Dairy Res., 49, 187-196.

LINDEN G., 1971. Application de la spectrophotométrie d'absorption atomique dans les laboratoires d'industrie alimentaire. Ind. Aliment. Agric., 6, 793-803.

Muir D.D., SweEtsur A.W.M., 1976. The influence of naturally occuring levels of urea on the heat stability of bulk milk. J. Dairy Res., 43, 495-499.

MUIR D.D., SwEETSUR A.W.M., 1977. Effect of urea on the heat coagulation of caseinate complex in skim milk. J. Dairy Res., 44, 249-257.

MUlLeR L.L., 1965. Milk powders for recombined products. Supplement of Aust. J. Dairy Technol., September 1965, 49-53.

Rose D., 1961. Factors affecting the $\mathrm{pH}$ sensitivity of the heat stability of milk from individual cows. J. Dairy Sci., 44, 1405-1413.

Rose D., 1962. Factors affecting the heat stability of milk. J. Dairy Sci., 45, 1305-1311.

SAINCLIVIER M., 1959. Etude des conditions d'emploi de la méthode Smeets pour le dosage du calcium ionisé dans un ultrafiltrat de lait. Ann. Technol., II, $155-170$.

Serres L., Amariglio S., Pertransxiene D., 1973. Contrôle de la qualité des produits laitiers. Tome 1, Ministère de l'Agriculture, Direction des Services Vétérinaires, Paris.

SWEeTSuR A.W.M., White J.C.D., 1974. Studies on the heat stability of milk protein. I. Interconversion of type A and type B milk heat stability curves. J. Dairy Res., 41, 349-358.

Sweetsur A.W.M., White J.C.D., 1975. Studies on the heat stability of milk protein. III. Effect of heat induced acidity in milk. J. Dairy Res., 42, 73-88.

Weeb B.H., Johnson A.H., ALFoRd J.A., 1978. Fundamentals of Dairy Chemistry. Second edition, the AVI Publishing Company, Inc. Wesport, Connecticut. 